\title{
Effects of medio-cortical frontal lesions on DRL performance in the rat
}

\author{
ROBERT NUMAN \\ Department of Pharmacology and Toxicology, University of Rhode Island, Kingston, Rhode Island 02881
}

and

\author{
A. RONALD SEIFERT and JOEL F. LUBAR \\ University of Tennessee, Knoxville, Tennessee 37916
}

\begin{abstract}
Damage to medial frontal cortex impaired the performance of rats during DRL 20 acquisition. This deficit was diminished when a feedback stimulus signaled the termination of the required delay. CRF response rates were not affected by frontal damage. It is noted that previous investigations have demonstrated DRL deficits following frontal damage in cat and monkey, but not in rat when frontal-polar cortex is removed. Our results, therefore, support the view that medial frontal cortex, rather than frontal-polar cortex, is the rat homologue to frontal association cortex of more advanced species. The relationship between fronto-limbic connections and response regulation is discussed.
\end{abstract}

Damage to frontal association cortex in monkeys (Glickstein, Quigley, \& Stebbins, 1964) and cats (Numan \& Lubar, 1974) leads to impaired performance on DRL (differential reinforcement of low rates) schedules of reinforcement. In contrast to these findings for monkey and cat, Schmaltz and Isaacson (1968) were unable to uncover such a deficit in rats following damage to the frontal poles. This discrepancy in rat may have anatomical roots. Recent anatomical (Leonard, 1969) and behavioral (Divac, 1971) investigations have suggested that medial frontal cortex, rather than frontal-polar cortex, is the rat homologue to frontal association cortex of more advanced species. Therefore, we undertook the present experiment in order to assess the effects of anterior medio-cortical damage in rats on DRL performance.

\section{METHOD}

\begin{abstract}
Subjects
Male hooded rats of the Long-Evans strain, weighing between 225 and $250 \mathrm{~g}$ at the beginning of the experiment, were used. The animals were housed individually under standard laboratory conditions. Water was always freely available, while food intake was restricted as indicated below.
\end{abstract}

\section{Surgery and Histology}

The subjects were randomly assigned to one of two groups. Nine rats received bilateral removal of medial frontal cortex, and 11 rats served as sham-operated controls. All surgery was performed under

The research reported in this paper was supported by Grant MH-14182-06 from the United States Public Health Service. We wish to thank Renee Culver and Everett Magagnos for their technical assistance, and Kathleen McGovern and Jean Mackey for help in preparing the manuscript. Requests for reprints should be addressed to Robert Numan, Department of Pharmacology, University of Rhode Island, Kingston, Rhode Island 02881.
Nembutal anesthesia. Animals were placed in a stereotaxic instrument, and an area of skull, extending from bregma to the frontal poles and $2 \mathrm{~mm}$ lateral on each side of the midline, was removed. At this point, surgical intervention was discontinued for the sham-operated subjects. For the frontal subjects, the dura was cut and medial frontal cortex, as defined by Leonard (1969), was removed by aspiration. Special care was taken so as not to inflict damage to the superior saggital sinus. All wounds were packed with gelfoam, and the skin was sutured closed. An antibiotic was administered to all subjects following surgery.

Following behavioral testing, the frontal subjects received a lethal dose of Nembutal and were perfused intracardially with normal saline followed by a $10 \%$ Formalin in saline solution and the brains were removed. The brains were then frozen and sections cut at $30 \mu$ through the extent of the lesion. Every fifth section was mounted and stained with cresyl violet.

\section{Apparatus}

All experimental testing was carried out in sound-attenuated operant chambers supplied with ventilation fans. Reinforcement consisted of a 45-mg Noyes food pellet. All sessions were $30 \mathrm{~min}$ in length.

Several investigators (Ellen \& Aitken, 1971; Kelsey \& Grossman, 1971) have suggested that external noises associated with the operation of programming equipment can provide cues for subjects performing on DRL schedules. We, therefore, took special precautions to eliminate these factors. All schedule contingencies and recordings were carried out with soundless solid-state circuitry located in a room several meters and four walls removed from the room which housed the operant chambers.

\section{Presurgical Testing}

Prior to surgery, all subjects were placed on a $22-\mathrm{h}$ food-deprivation schedule. When body weights stabilized at about $85 \%$ of free-feeding weight, the subjects were shaped to press a lever for food reinforcement on a schedule of continuous reinforcement (CRF). Shaping was terminated when the animals achieved a criterion of at least 40 responses in $30 \mathrm{~min}$ : After reaching this criterion, the animals were run for an additional 6 days on CRF. Surgery was performed on the day following presurgical CRF training.

\section{Postsurgical Testing}

Following surgery, all animals were allowed a 1-week recovery period in which food and water were freely available. Following 
recovery, food deprivation was reinstated. When body weights again stabilized at about $85 \%$ of free-feeding weight, 6 additional days of CRF trials were run.

On the day after completion of CRF, the animals were placed on a differential reinforcement of low rates 20-sec schedule (DRL 20). On this schedule, responses are reinforced only if they occur at least $20 \mathrm{sec}$ following a prior response. If successive responses occur less than $20 \mathrm{sec}$ apart, the DRL timer is recycled, and the animal must withhold responding for an additional $20 \mathrm{sec}$ before a response will lead to reinforcement.

DRL testing was carried out in three successive stages. First, acquisition training was carried out for 30 days. During this stage, there were no exteroceptive stimuli available to signal the end of the required delay. Following acquisition, 15 days of DRL 20 with feedback was instituted. Under this condition, a cue light, located above the lever, was turned on at the end of the 20-sec DRL interval. Any responses occurring while the cue light was on led to reinforcement, turned off the light, and recycled the DRL timer. The feedback condition was followed by 15 days of feedback withdrawal, during which the cue light was no longer utilized.

Throughout the entire experiment, the animals were tested for $30 \mathrm{~min}$ each day, 7 days per week. The following events were recorded, and represent the main experimental variables: reinforcements, responses, and percent efficiency (reinforcements/ responses $x$ 100). All statistical comparisons were calculated with $t$ test, two-tailed.

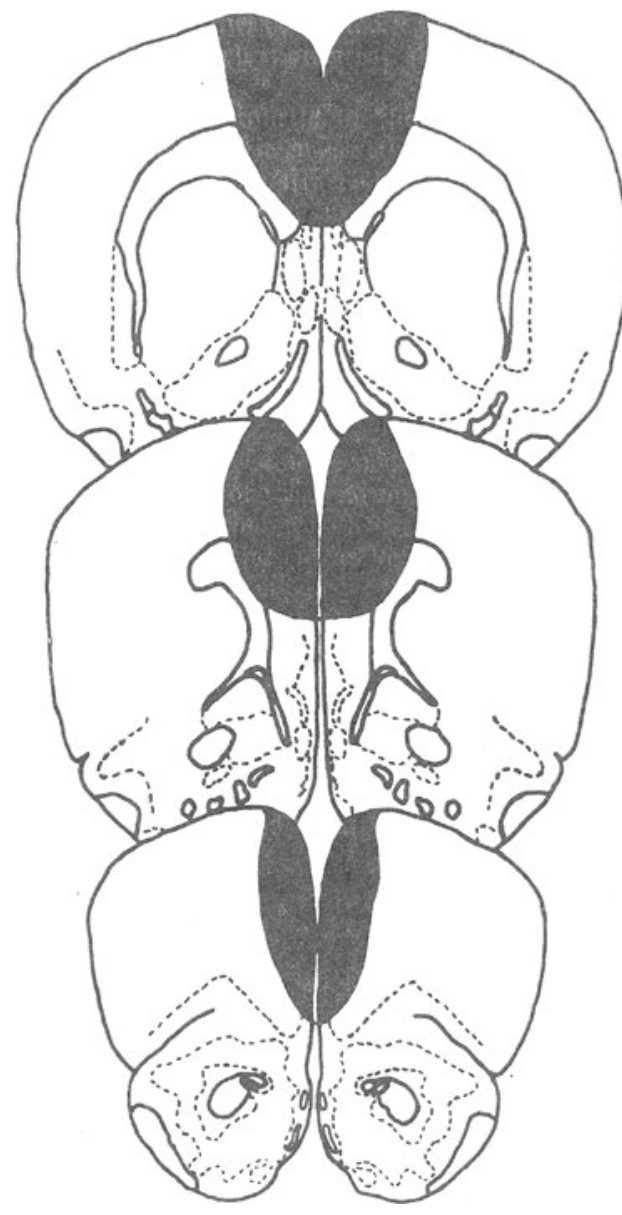

9.0

10.0

Figure 1. Reconstruction of representative lesion in medial frontal cortex. Numbers at the right of each section refer to A-P coordinates from the Pellegrino and Cushman (1967) atlas.

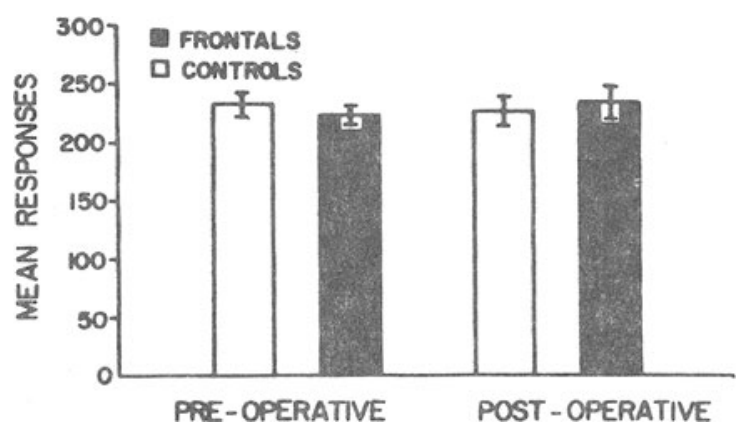

Figure 2. Mean number of responses $( \pm$ S.E.) during the 6-day periods of preoperative and postoperative CRF.

\section{RESULTS}

\section{Histology}

In all frontal operated rats, most of the medial frontal cortex, as defined by Leonard (1969), was removed. In general, the lesions removed cortex from the medial portions of the hemisphere anterior to the genu of the corpus callosum. Most subjects also received some damage to the corpus callosum. None of the lesions damaged olfactory or striatal regions. A reconstruction of a representative lesion, based on the atlas of Pellegrino and Cushman (1967), is shown in Figure 1.

\section{CRF}

Figure 2 shows the mean number of responses emitted for both groups during the 6-day periods of presurgical and postsurgical CRF. There were no significant differences between the groups either preoperatively or postoperatively. Within-groups comparisons were also insignificant; neither sham operation nor frontal damage produced changes in CRF response rates.

\section{DRL Acquisition}

Figure 3 shows the mean percent efficiency, mean reinforcements, and mean responses for the two groups over the 30-day acquisition period. While there is little difference between the groups during the initial stages of DRL acquisition, the performance curves gradually diverge on subsequent days. While the sham operates improve on all measures of DRL performance, no such improvement is seen for the frontal subjects. By the end of DRL acquisition, frontal subjects performed less efficiently, emitted more responses, and received fewer reinforcements than the sham-operated controls. A summary of the statistical comparisons between frontals and controls on all measures of DRL performance throughout the entire period of acquisition is presented in Table 1 .

\section{Feedback and Withdrawal}

The effect of the feedback stimulus (indicating the availability of reinforcement) is clear (Figure 4). Both 


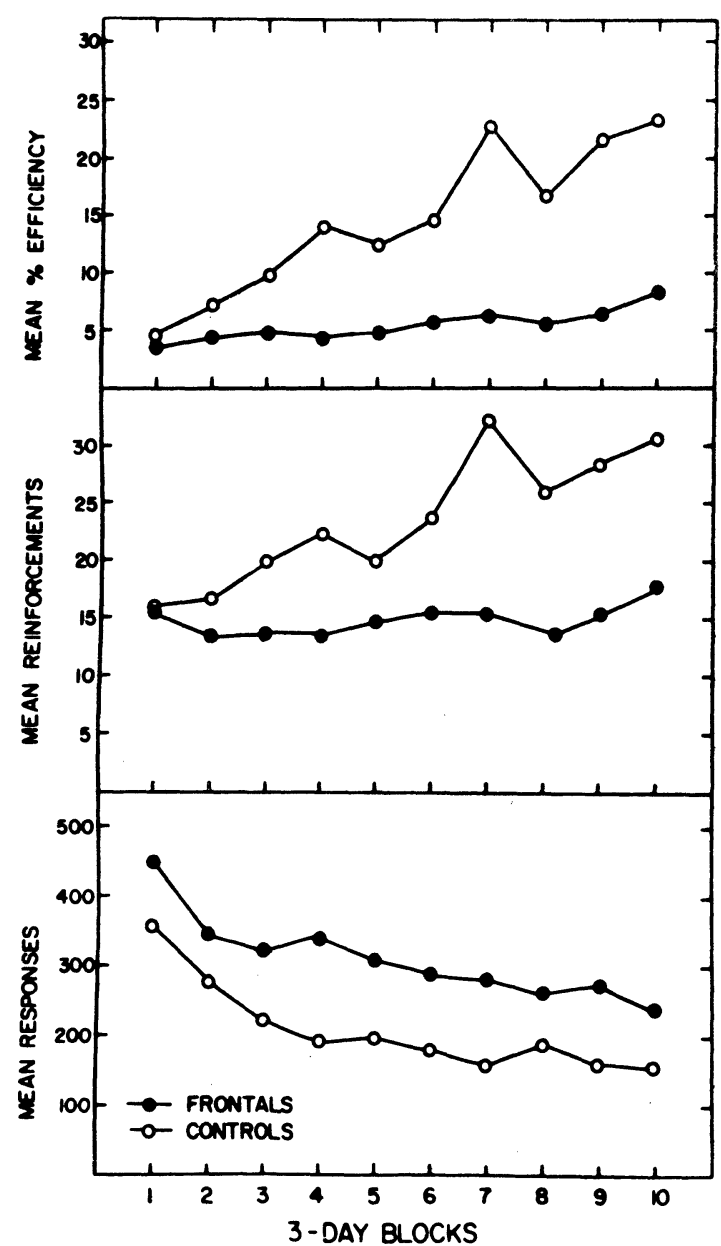

Figure 3. Mean percent efficiency, reinforcements, and responses during DRL acquisition.

groups increased their efficiency and number of reinforced responses while reducing their response rates. While frontal subjects are still performing poorly during the initial stages of feedback training, they improve rapidly over days. There were no significant differences between the groups on any measure of performance during the last 9 days of feedback testing. The feedback stimulus, therefore, ameliorated the deficit on DRL evident during acquisition in frontal subjects.

During the withdrawal period, both groups performed better than during acquisition, but less well in comparison to their performance during feedback. There were no significant differences between the groups on any measure of DRL performance throughout the entire withdrawal period.

\section{Extent of Cortical Removal and the DRL Impairment}

While there was not a clear-cut relationship between overall lesion size and behavioral impairment during DRL acquisition, the locus of the frontal
Table 1

Summary of Statistical Comparisons Between Frontals and Controls on All Measures of DRL Performance Throughout Acquisition

\begin{tabular}{|c|c|c|c|c|c|c|c|c|c|c|}
\hline \multirow{2}{*}{$\begin{array}{l}\text { Performance } \\
\text { Measure } \\
\end{array}$} & \multicolumn{4}{|c|}{ Successive 3-Day } & Block & \multicolumn{5}{|c|}{ During Acquisition } \\
\hline & 1 & 2 & 3 & 4 & 5 & 6 & 7 & 8 & 9 & 10 \\
\hline Efficiency & NS & NS & S & S & NS & S. & $S$ & $\mathbf{S}$ & $S$ & $S$ \\
\hline Reinforcements & NS & NS & MS & S & NS & MS & S & S & MS & MS \\
\hline Responses & $\mathbf{S}$ & MS & S & S & $\mathrm{S}$ & S & $\mathbf{S}$ & $\mathbf{S}$ & $\mathbf{S}$ & $\mathbf{S}$ \\
\hline
\end{tabular}

Note $-S=$ significant difference, at least $p<.05 . M S=$ marginally significant, $.1>p>.05 . N S=$ not significant, $p>.1$. All comparisons made with t test, two tailed.

damage did appear to be of some importance. A histological-behavioral analysis on individual animals suggested two general trends in this regard. First, the greater the extent of medial cortical damage along the dorso-ventral axis, the greater the DRL impairment. Second, subjects with lesions which included removal of cortical tissue posterior to A-P 10.0 tended to show greater impairments during DRL acquisition than subjects whose lesions were restricted to cortical tissue anterior to A-P 10.0 (see Figure 1). Subjects with

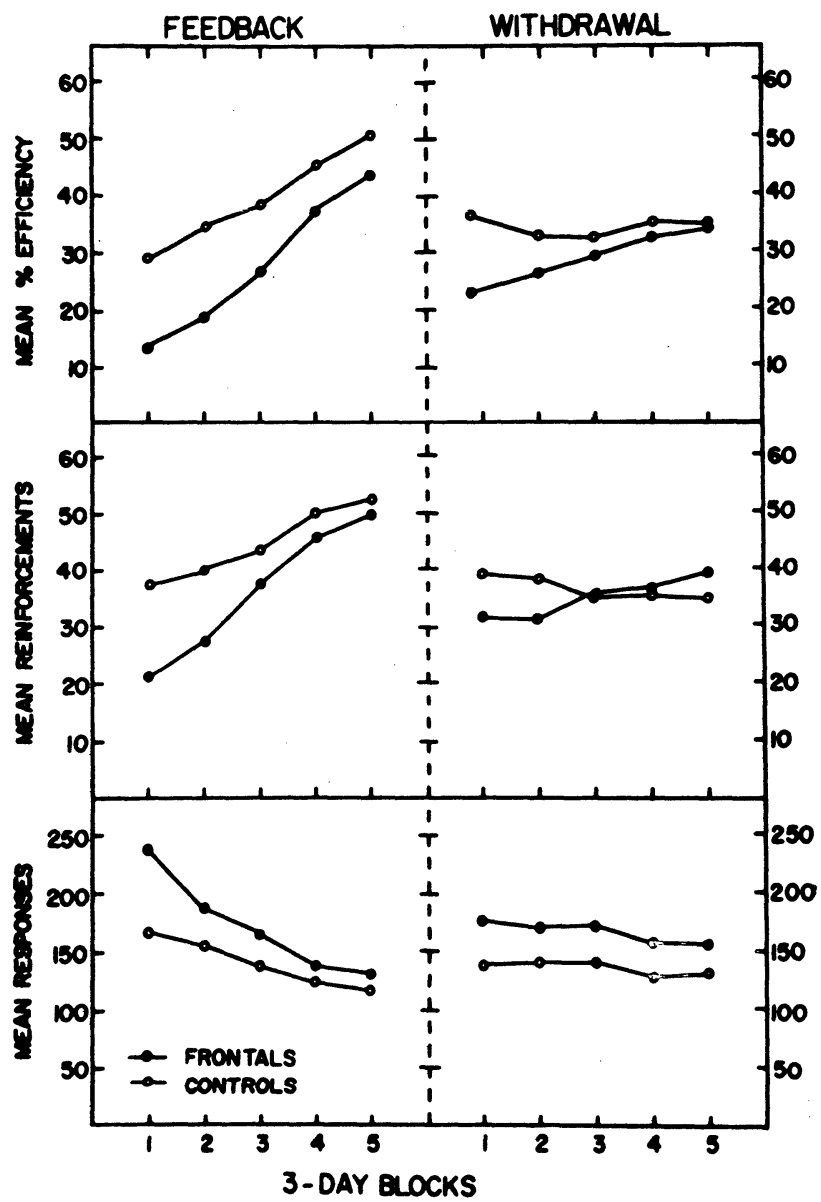

Figure 4. Mean percent efficiency, reinforcements, and responses during feedback and feedback withdrawal. 
lesions which included damage to these two areas were clearly deficient during acquisition when compared with controls. While subjects with more restricted lesions showed performance patterns which overlapped somewhat with those of the least efficient control animals.

\section{DISCUSSION}

Previous studies have reported DRL impairment in cats (Numan \& Lubar, 1974) and monkeys (Glickstein, Quigley, \& Stebbins, 1964) following damage to frontal association cortex. In contrast to these effects, Schmaltz and Isaacson (1968) were unable to demonstrate such a deficit for rats following damage to the frontal poles. In reference to this latter finding, however, it should be noted that Leonard (1969), in an anatomical study, has suggested that medial cortex, anterior to the corpus callosum, rather than frontal-polar cortex is the rat homologue of frontal association cortex in more advanced species. The results of the present experiment are in support of Leonard's (1969) anatomical results. We found that damage to medial frontal cortex in rats disrupts the acquisition of a DRL operant in a like manner to that noted for more advanced species.

As in cats (Numan \& Lubar, 1974), the DRL impairment in frontal rats is ameliorated when the availability of reinforcement is signaled by a visual stimulus. This finding indicates that frontal rats, like frontal cats (Numan \& Lubar, 1974; Wagman, 1968) and monkeys (Stamm, 1970), may have difficulty regulating response tendencies in the absence of meaningful exteroceptive feedback to guide responding. On the other hand, these results do not fit well with a simple response disinhibitory effect of frontal damage, since response inhibition normalizes during feedback training. Moreover, our finding that frontal damage in the rat does not lead to increased responding for food reinforcement during CRF testing suggests that these results cannot be explained in terms of lesion-induced motivational changes.

In contrast to these findings during DRL acquisition and feedback, the frontal rat, unlike the frontal cat (Numan \& Lubar, 1974), is not impaired on DRL during feedback withdrawal. Whether this result in rat is due to a protracted training effect or some facilitative function of cue presentation cannot be determined from the present results. However, one can suggest that the DRL deficit in the frontal rat is less severe than that observed in frontal cats.

Our results are consistent with those reported recently by Divac (1971). He found that damage to medial frontal cortex disrupted spatial reversal learning in rats, while damage to the frontal poles did not. He also suggests that this impairment is less severe in rats than in cats and monkeys.

The relatively mild impairments in response regulation observed in rats when compared to cats and monkeys following frontal damage may have anatomical roots. The limbic system has long been known to subserve response regulatory functions (Kaada, 1951; Kimble, 1968; Lubar \& Numan, 1973; McCleary, 1966; Vanderwolf, 1971). In primates, frontal association cortex has numerous anatomical connections with limbic structures (Devito \& Smith, 1964; Johnson, Rosvold, \& Mishkin, 1968; Nauta, 1964; Van Hoesen, Pandya, \& Butters, 1972). However, Leonard (1969) could not substantiate many of these connections between frontal cortex and limbic system in rat. Further, recent anatomical findings by Voneida and Royce (1974) suggest that cat frontal cortex represents an intermediate level of frontolimbic organization, having more limbic connections than rat but less than monkey. Thus, anatomical results indicate a higher degree of fronto-limbic connectivity as we ascend the phylogenetic scale, and behavioral findings suggest a similar trend for the degree of response regulatory impairments observed following frontal damage.

In an attempt to explain the nature of the response regulatory impairments observed following frontal or limbic damage, Numan (1972) has formulated a model of response regulation based on fronto-limbic relations. In brief, frontal cortex is designated as the generator of motor programs. Following response initiation, the motor program is stored in hippocampus. Peripheral and central (corollary discharges) feedback, as a consequent of responding, is passed to the hippocampus for comparison with the stored motor program. This comparation process (of which hippocampal theta is seen as a correlate) then leads to either response termination (goal achievement) or the formation of new response modes in frontal cortex (error detection). This model fits fairly well with a large body of anatomical and behavioral data (see Lubar \& Numan, 1973; Numan, 1972; Numan \& Lubar, 1974, for a more complete description) and stresses the importance of fronto-limbic interrelations for behavioral flexibility.

\section{REFERENCES}

Devito, J. L., \& Smith, O. A., JR. Subcortical projections of the prefrontal lobe of the monkey. Journal of Comparative Neurology, 1964, 123, 413-424.

Divac, I. Frontal lobe system and spatial reversal in the rat. Neuropsychologia, 1971, 9, 175-183.

Ellen, P., \& Aitken, W. C., JR. A bsence of temporal discrimination following septal lesions. Psychonomic Science, 1971, 22, 129-131.

Glickstein, M., Quigley, W. A., \& Stebbins, W. C. Effect of frontal and parietal lesions on timing behavior in monkeys. Psychonomic Science, 1964, 1, 265-266.

Johnson, T. N., Rosvold, H. E., \& Mishkin, M. Projections from behaviorally-defined sectors of the prefrontal cortex to the basal ganglia, septum, and diencephalon of the monkey. Experimental Neurology, 1968, 21, 20-34. 
KA ADA, B. R. Somato-motor, autonomic and electrocorticographic responses to electrical stimulation of 'rhinencephalic' and other structures in primate. cat, and dog. Acta Physiologica Scandinavica, 1951, 24, Supplement 83, 1-285.

Kelsey. J. E.. \& Grossman, S. P. Nonperseverative disruption of behavioral inhibition following septal lesions in rats. Journal of Comparative and Physiological Psychology, 1971, 75, 302-311.

Kimble, D. P. The hippocampus and internal inhibition. Psychological Bulletin. 1968, 70. 285-295.

Leonard. C. M. The prefrontal cortex of the rat. I. Cortical projections of the medio dorsal nucleus. II. Efferent connections. Brain Research, 1969. 12. 321-343.

Lubar, J. F., \& Numan, R. Behavioral and physiological studies of septal function and related medial cortical structures. Behavioral Biology, 1973, 8, 1-25.

McCleary, R. A. Response modulating function of the limbic system. Initiation and suppression. In E. Stellar and J. M. Sprague (Eds.), Progress in physiological psychology (Vol. 1). New York: Academic Press. 1966. Pp. 210-266.

Nauta, W. J. H. Some efferent connections of the prefrontal cortex in the monkey. In J. M. Warren \& K. Akert (Eds.), The frontal granular cortex and behavior. New York: McGraw-Hill, 1964. Pp. 397-409.

Numan, R. The effects of frontal and septal ablation on response regulution in the cat. Unpublished doctoral dissertation, University of Tonnessee, Knoxville, Tennessee. 1972.
Numan. R., \& Lubar, J. F. Role of the proreal gyrus and septal area in response modulation in the cat. Neuropsychologia. 1974, 12, 219-234.

Pellegrino, L. C., \& Cushman, A. J. A stereotaxic atlas of the rat brain. New York: Appleton-Century-Crofts, 1967.

Schmaltz, L. W.. \& Isaacson, R. L. Effects of caudate and frontal lesions on retention and relearning of a DRL schedule. Journal of Comparative and Physiological Psychology, 1968, 65. 343-348.

Stamm, J. S. Dorsolateral frontal ablations and response processes in monkeys. Journal of Comparative and Physiological Psychology', 1970, 70, 437.447.

VANDERWOLF, C. H. Limbic-diencephalic mechanisms of voluntary movement. Psychological Review, 1971, 78, 83-113.

Van Hoeson, W. G., Pandya, D. N., \& Butters, N. Cortical afferents to the entorhinal cortex of the rhesus monkey. Science, 1972, 175, 1471-1473.

Voneida, T. j., \& Royce, G. J. Ipsilateral connections of the gyrus proreus in the cat. Brain Research, 1974, 76, 393-400.

Wagman, A. M. I. The effect of frontal lobe lesions upon behavior requiring use of response-produced cues. Journal of Comparative and Physiological Psychology, 1968, 66. 69-76.

(Received for publication April 16, 1975; revision received May 19, 1975.) 